Supporting Information : Bulk-Induced Flicker Noise at the Surface of Three-Dimensional Topological Insulators

Semonti Bhattacharyya ${ }^{1}$, Mitali Banerjee ${ }^{1}$, Hariharan Nhalil ${ }^{1}$, Saurav

Islam $^{1}$, Chandan Dasgupta ${ }^{1}$, Suja Elizabeth ${ }^{1}$, Arindam Ghosh ${ }^{1}$

${ }^{1}$ Department of Physics, Indian Institute of Science, Bangalore 560 012, India 


\section{I.CRYSTAL STRUCTURE OF BSTS}

Fig. S1 shows the powder XRD pattern of BSTS. The pattern is refined and indexed in rhombohedral structure with R-3m space group. The refined lattice parameters are $a=4.243(1) \AA$ and $c=29.812(8) \AA$ which are in agreement with the reported values [1]. The inset of Fig. S1 shows a magnified view of (107) and (0012) peaks which are characteristic of BTS type chalcogen ordering $[1,2]$. This implies that, chalcogen ordering is preserved to some extent in spite of significant change in stoichiometry. Fig. S2 gives the XRD pattern along (111) crystallographic plane ((001) plane in the hexagonal setting) of a cleaved single crystal. Several $00 L$ reflections are observed obeying the systematic absence for the rhombohedral space group.

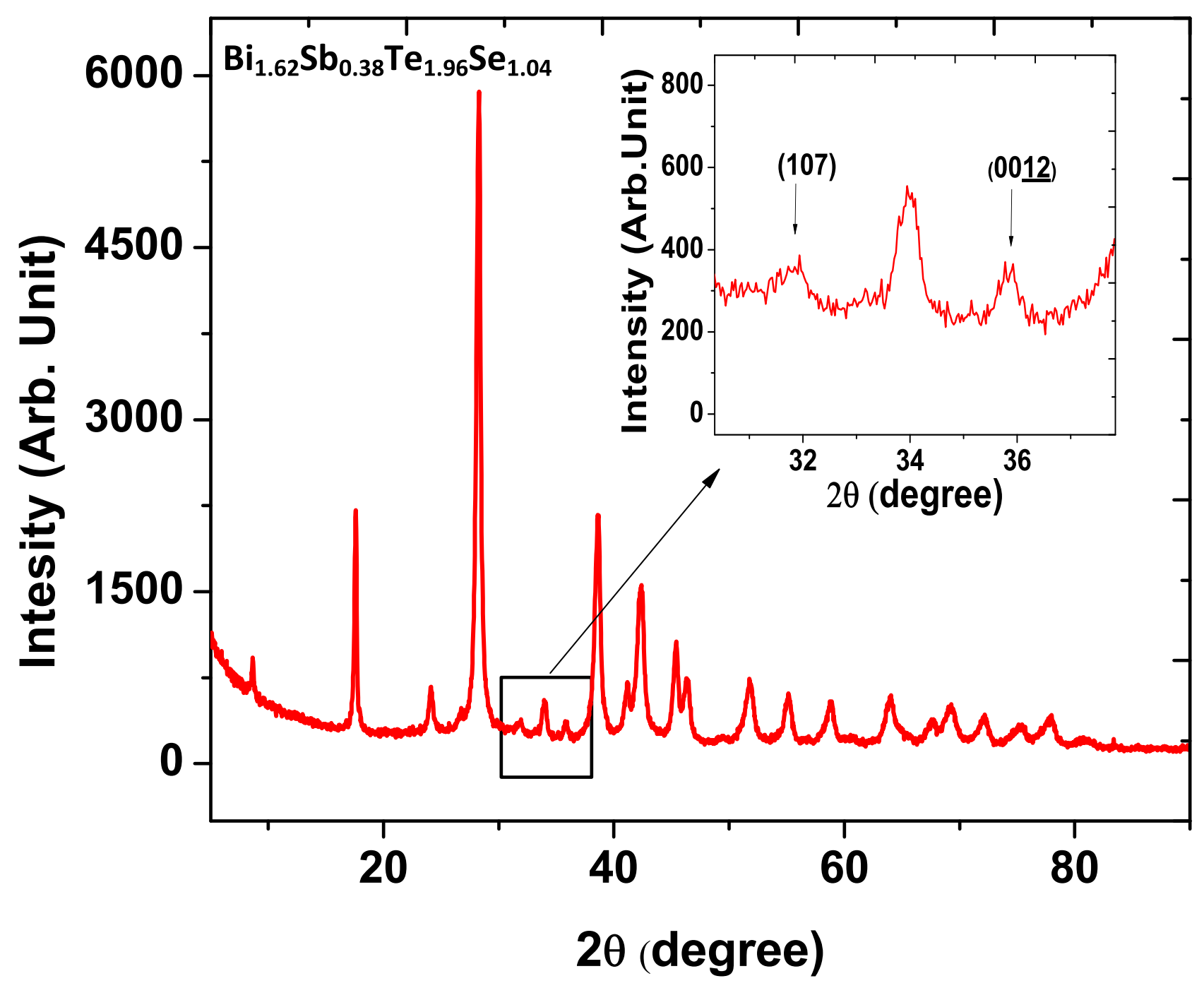

Supplementary Figure S1 : Powder XRD pattern of crushed single crystals of BSTS. Inset shows a magnified view of the (107) and (0012) peaks. 


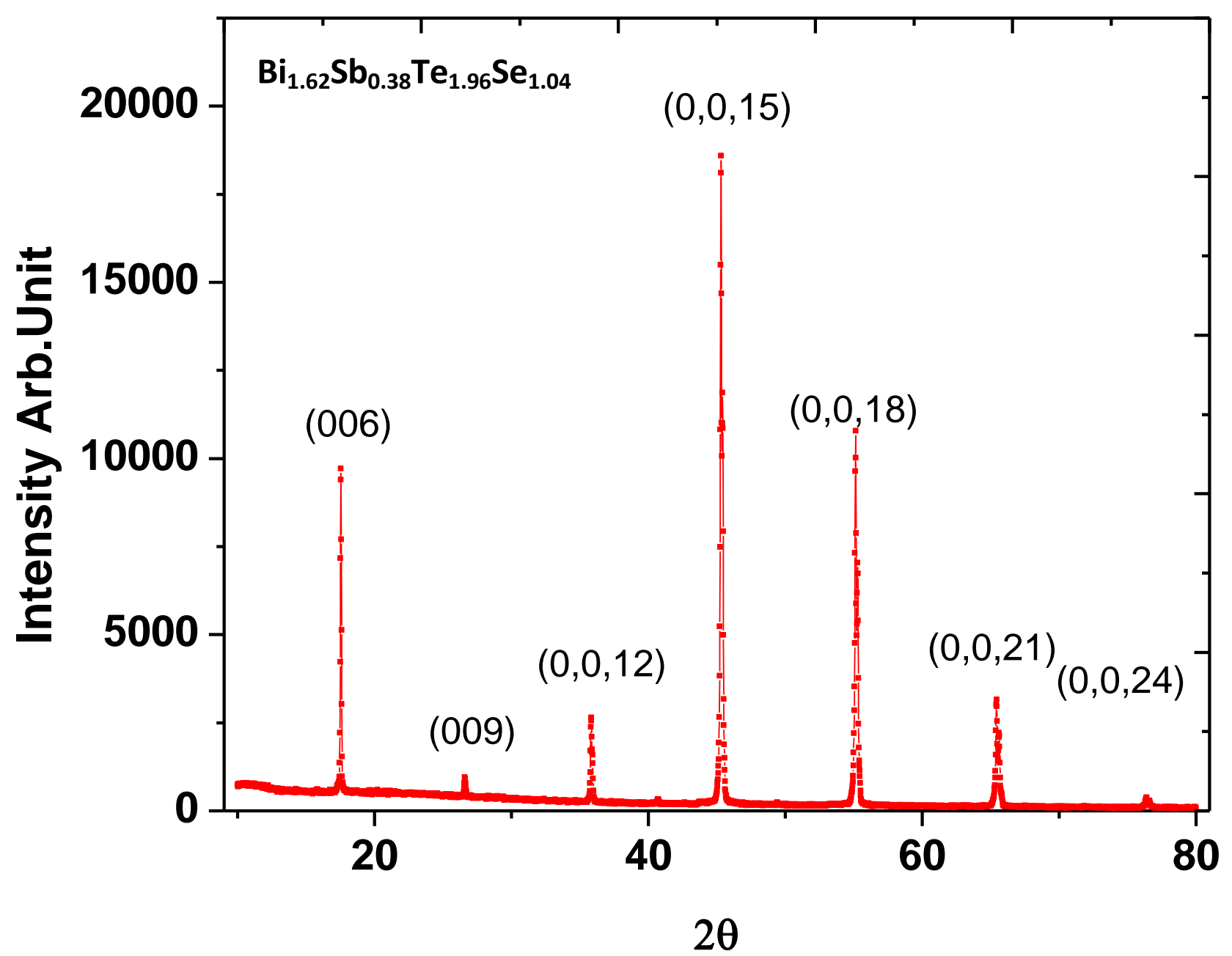

Supplementary Figure S2 : Single crystal XRD pattern of the cleaved single crystal of BSTS . Clear $00 L$ reflections when $L=3 n$ are observed and indexed. 


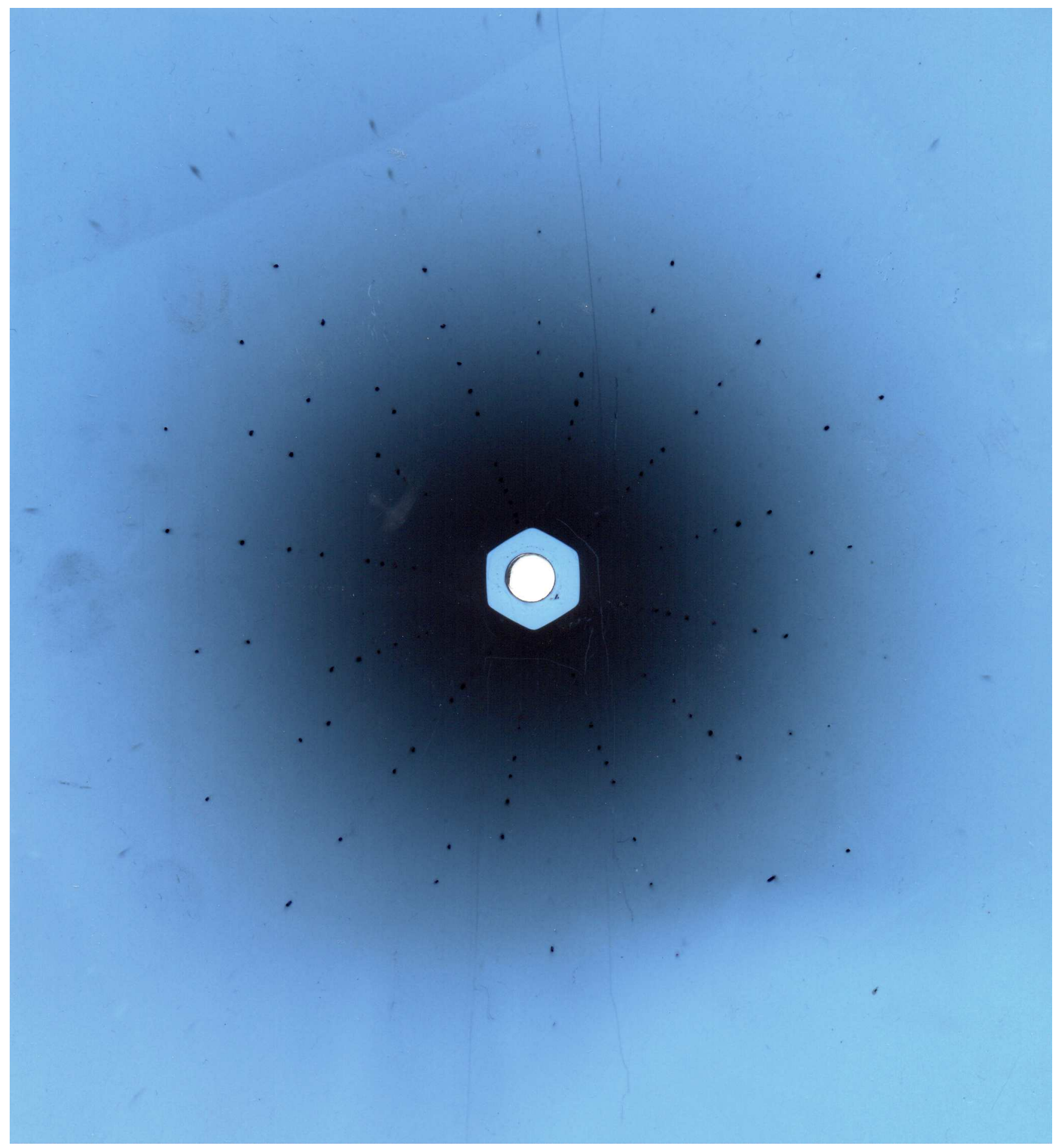

Supplementary Figure S3 : Single crystal reflection Laue photograph using Cu Ka x-rays.Sharp spots and symmetry confirms good single crystalline nature. 


\section{II.COMPOSITION ANALYSIS}

Composition of the as-grown crystal was analyzed using JEOL JXA-8530F field emission electron probe micro analyzer with incident beam voltage of $15 \mathrm{kV}$ and current of $50 \mathrm{nA}$ in the wavelength dispersion mode. Three single crystal samples cleaved from different parts of the grown crystal were used in these studies. Sample surfaces were cleaned thoroughly before the studies and five or more different spots were scanned on each sample. Results of the studies are summarized in TABLE S1. The composition thus determined are consistent with nominal values of each sample and we could not detect any impurities within the detection limit of EPMA. The EDS spectrum acquired is shown in Fig. S4.

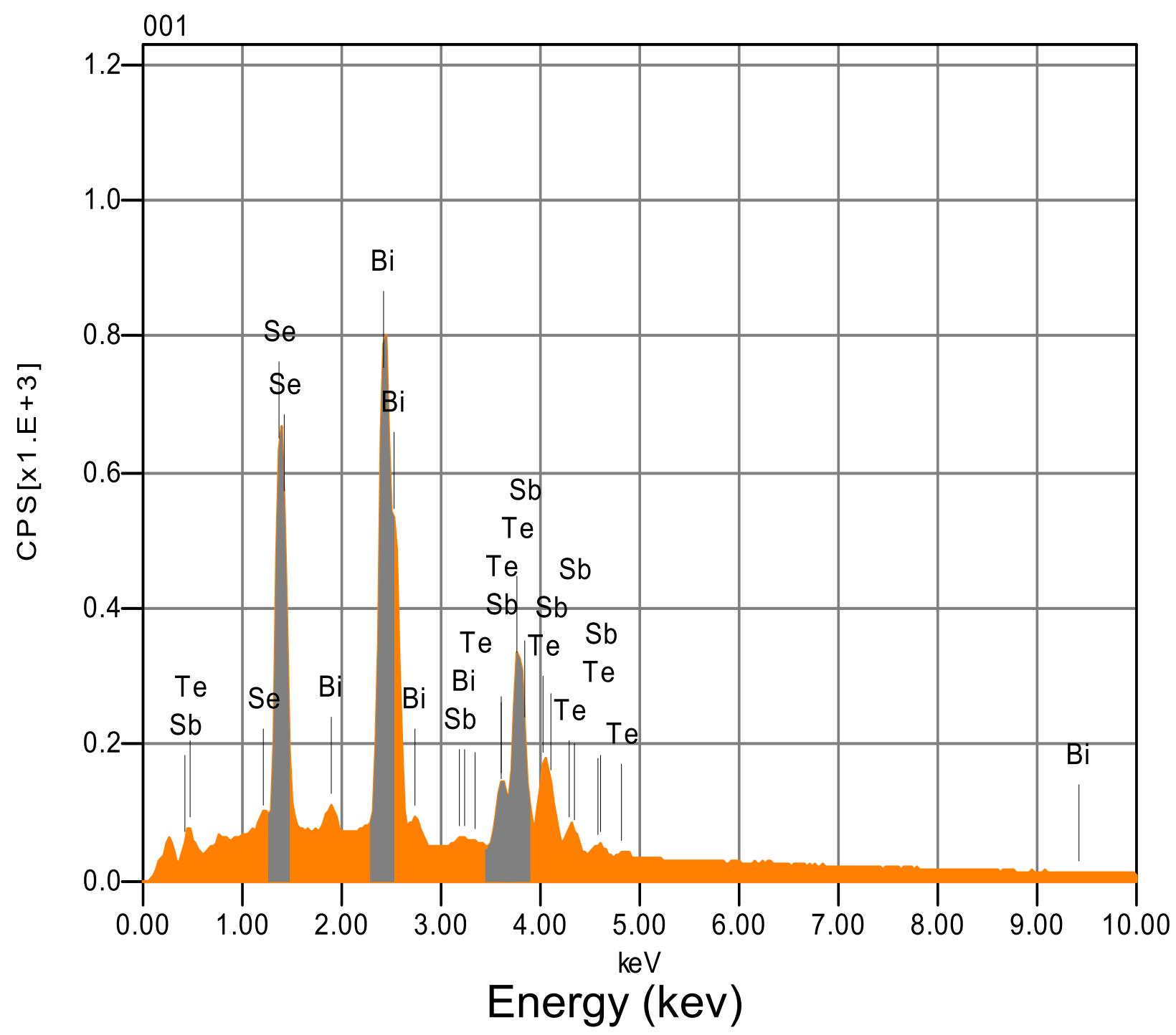

Supplementary Figure S4 . EDS spectrum acquired from the cleaved BSTS single crystal. 


\begin{tabular}{|c|c|c|c|c|c|}
\hline Sample & Elements & Mass $\% \pm \sigma_{r}$ & Atomic $\% \pm \sigma_{r}$ & $\sigma_{r}(\%)$ & Estimated Composition \\
\hline \multirow{4}{*}{1} & $\mathrm{Bi}$ & $46.57 \pm 0.12$ & $31.20 \pm 0.08$ & 0.27 & \multirow{4}{*}{$\mathrm{Bi}_{1.61} \mathrm{Sb}_{0.39} \mathrm{Te}_{1.91} \mathrm{Se}_{1.09}$} \\
\hline & $\mathrm{Sb}$ & $10.42 \pm 0.04$ & $11.99 \pm 0.04$ & 0.36 & \\
\hline & $\mathrm{Te}$ & $28.80 \pm 0.05$ & $31.60 \pm 0.06$ & 0.19 & \\
\hline & $\mathrm{Se}$ & $14.23 \pm 0.02$ & $25.22 \pm 0.05$ & 0.20 & \\
\hline \multirow{4}{*}{2} & $\mathrm{Bi}$ & $45.81 \pm 0.12$ & $30.41 \pm 0.08$ & 0.27 & \multirow{4}{*}{$\mathrm{Bi}_{1.60} \mathrm{Sb}_{0.40} \mathrm{Te}_{1.94} \mathrm{Se}_{1.06}$} \\
\hline & $\mathrm{Sb}$ & $11.40 \pm 0.04$ & $12.99 \pm 0.04$ & 0.36 & \\
\hline & $\mathrm{Te}$ & $27.76 \pm 0.05$ & $30.19 \pm 0.06$ & 0.19 & \\
\hline & Se & $15.03 \pm 0.02$ & $26.01 \pm 0.05$ & 0.19 & \\
\hline \multirow{4}{*}{3} & $\mathrm{Bi}$ & $46.66 \pm 0.12$ & $30.99 \pm 0.08$ & 0.27 & \multirow{4}{*}{$\mathrm{Bi}_{1.63} \mathrm{Sb}_{0.37} \mathrm{Te}_{1.96} \mathrm{Se}_{1.04}$} \\
\hline & $\mathrm{Sb}$ & $11.15 \pm 0.04$ & $12.72 \pm 0.04$ & 0.36 & \\
\hline & $\mathrm{Te}$ & $26.83 \pm 0.05$ & $29.20 \pm 0.05$ & 0.19 & \\
\hline & Se & $15.40 \pm 0.03$ & $27.09 \pm 0.05$ & 0.19 & \\
\hline
\end{tabular}

Table S1. Results of WDS quantitative composition analysis of three BSTS samples . 
III. ATOMIC FORCE MICROSCOPIC IMAGES OF TI DEVICES

(a)

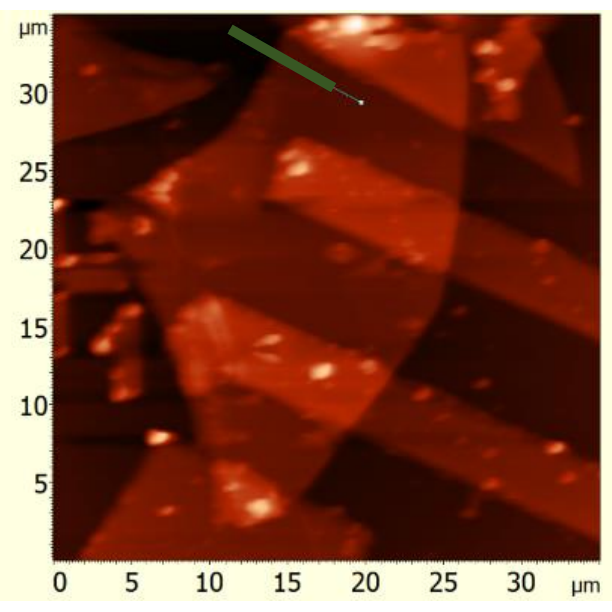

(c)

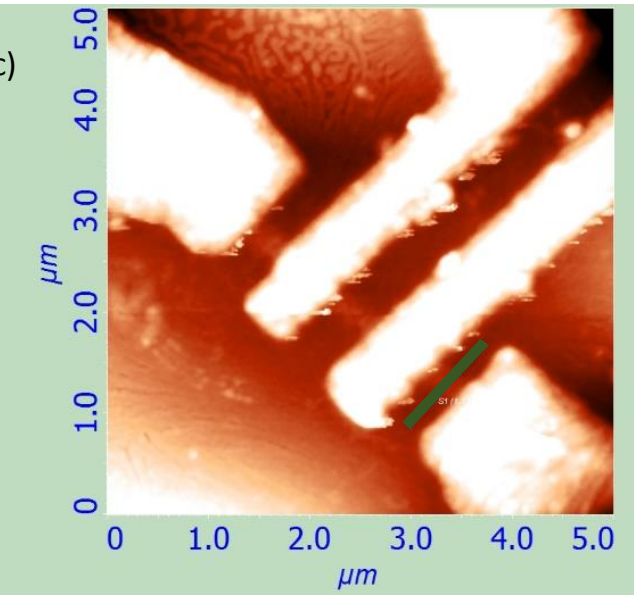

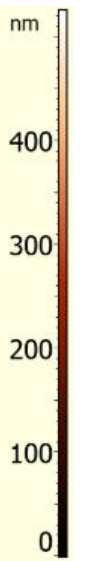
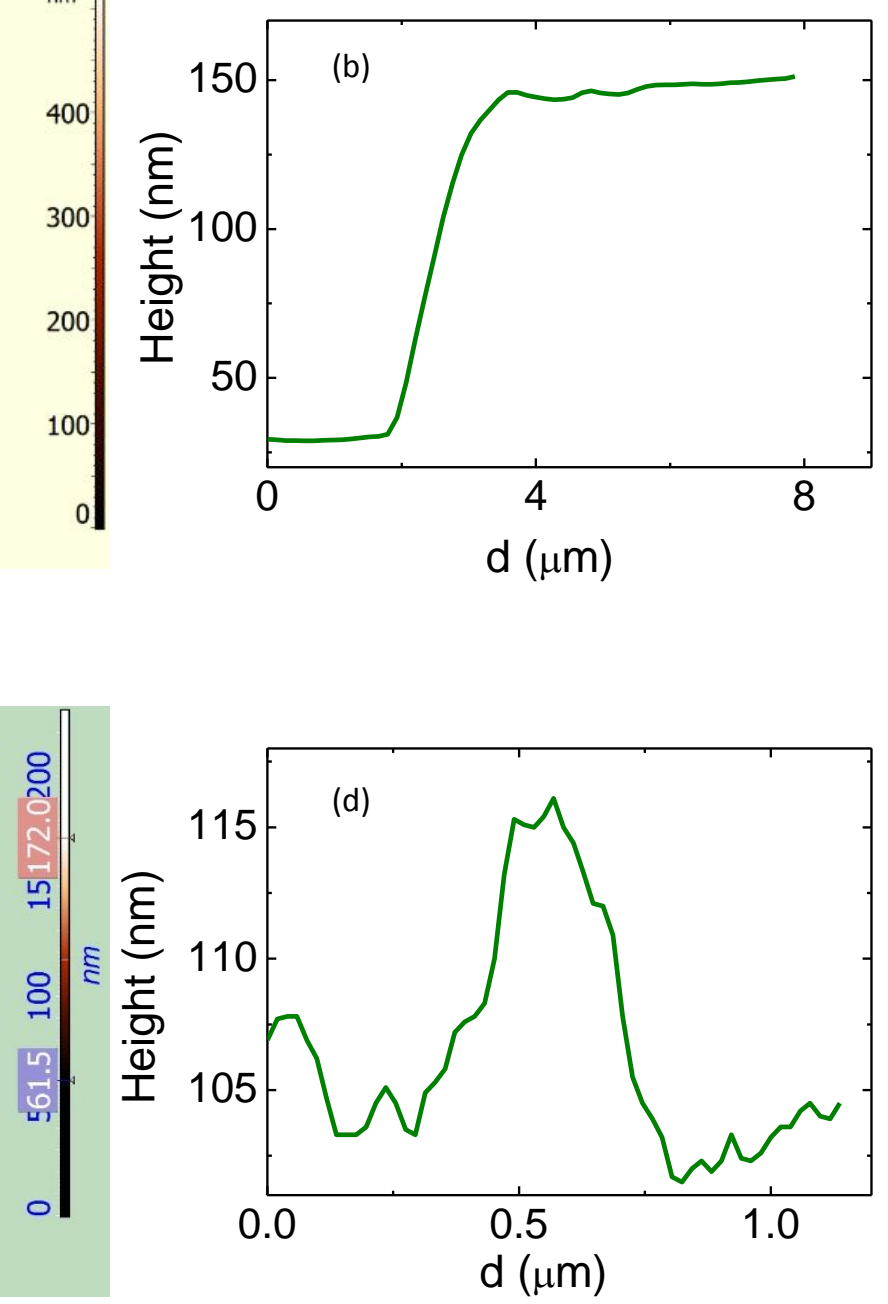

(e)

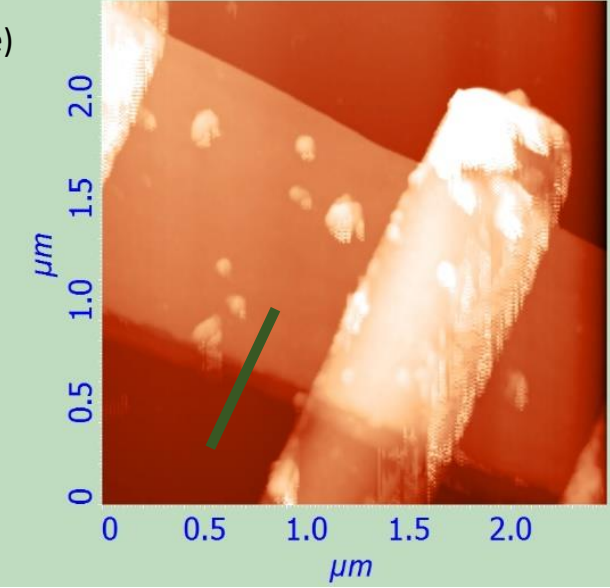

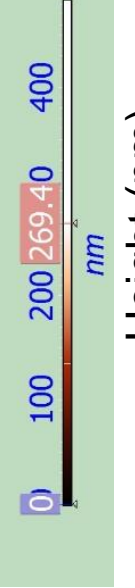

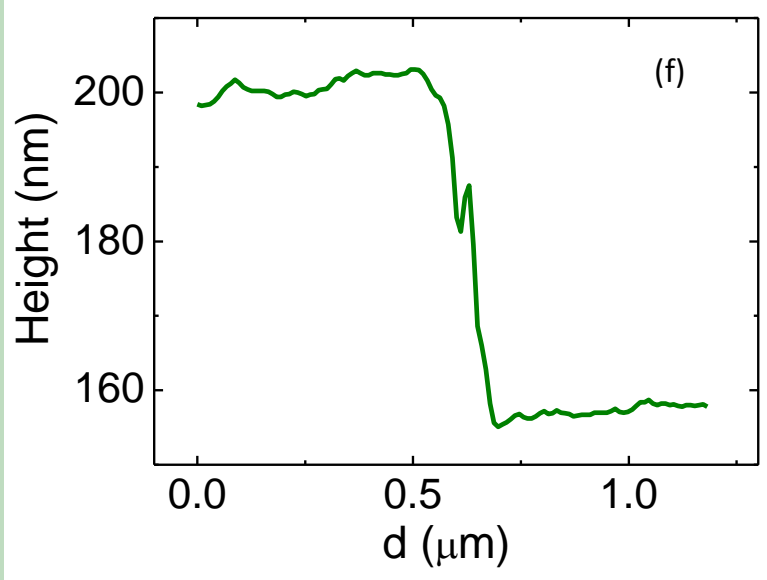

Supplementary Figure S5. AFM topography images of D110 (a), D10 (c) and D50 (e) and height profile of D110 (b), D10 (d), D50 (f) along the green line shown in the topography image. 
IV. $\mathbf{R}_{s}$ vs T FOR D10

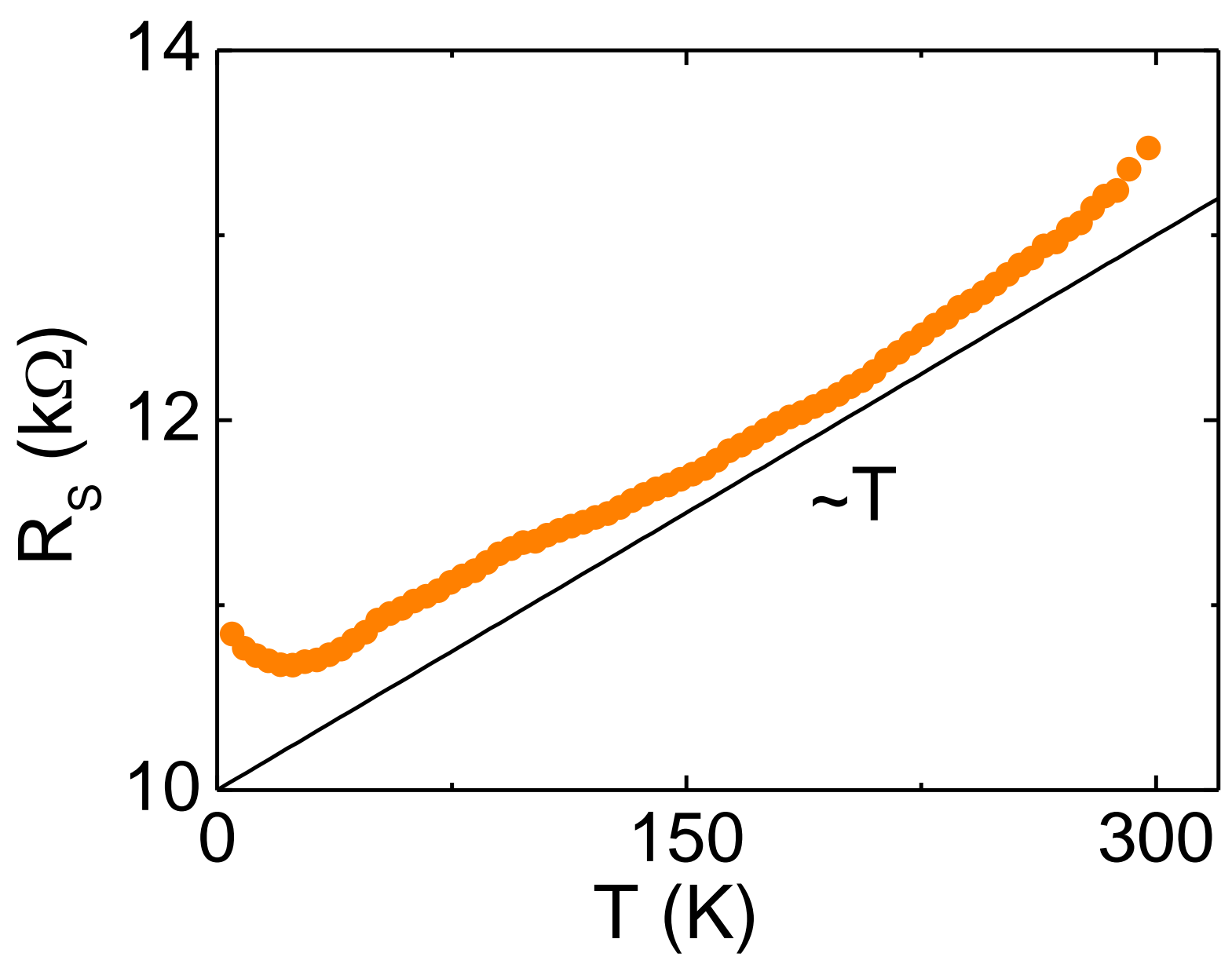

Supplementary Figure S6. Sheet-resistance $\left(\mathrm{R}_{S}\right)$ profile versus temperature $(T)$ in linear scale showing linear $T$-dependence of $\mathrm{R}_{S}$ in sample $\mathrm{D} 10$.

\begin{tabular}{|l|c|c|c|c|c|c|}
\hline $\begin{array}{l}\text { Sample } \\
\text { name }\end{array}$ & Substrate & $\begin{array}{c}\text { Length } \\
(L)(\mu \mathrm{m})\end{array}$ & $\begin{array}{c}\text { Width } \\
(W)(\mu \mathrm{m})\end{array}$ & $\begin{array}{c}\text { Thickness } \\
(d)(\mu \mathrm{m})\end{array}$ & $\begin{array}{c}\text { Area } \\
(A)\left(\mu \mathrm{m}^{2}\right)\end{array}$ & $\begin{array}{c}\text { Resistance @ 260 } \\
\mathrm{K}\left(R_{260}\right) \\
(\Omega)\end{array}$ \\
\hline $\mathrm{BLK1}{ }^{*}$ & $\mathrm{no}$ substrate & 2850 & 1590 & 80 & $453.15 \times 10^{4}$ & 0.45 \\
\hline $\mathrm{D} 1200$ & $\mathrm{SiO}_{2} / \mathrm{Si}^{++}$ & 96.1 & 445 & 1.2 & $4.27 \times 10^{4}$ & 26 \\
\hline $\mathrm{D} 1000$ & $\mathrm{SiO}_{2} / \mathrm{Si}^{++}$ & 108 & 265 & 1 & $2.86 \times 10^{4}$ & 52 \\
\hline $\mathrm{D} 580$ & $\mathrm{SiO}_{2} / \mathrm{Si}^{++}$ & 51.1 & 98.9 & 0.58 & $5.05 \times 10^{3}$ & 62 \\
\hline $\mathrm{D} 400$ & $\mathrm{SiO}_{2} / \mathrm{Si}^{++}$ & 17.7 & 60.3 & 0.4 & $1.07 \times 10^{3}$ & 272 \\
\hline $\mathrm{D} 110$ & $\mathrm{SiO}_{2} / \mathrm{Si}^{++}$ & 4.38 & 9.2 & 0.11 & 40.3 & 325.5 \\
\hline $\mathrm{D} 50$ & $\mathrm{SiO}_{2} / \mathrm{Si}^{++}$ & 1.43 & 1.5 & 0.05 & 2.14 & 798 \\
\hline $\mathrm{D} 10$ & $\mathrm{SiO}_{2} / \mathrm{Si}^{++}$ & 0.8 & 0.3 & 0.01 & 0.24 & $34.3 \times 10^{3}$ \\
\hline
\end{tabular}

${ }^{*}$ This sample was mechanically exfoliated from a big crystal and measured as a free standing sample without any substrate.

Table S2. Geometrical and electrical parameters of all 3D TI devices used for noise measurements. 
V. $\mathbf{R}_{s}$ and $\rho_{3 D}$ as a function of sample thickness $d$

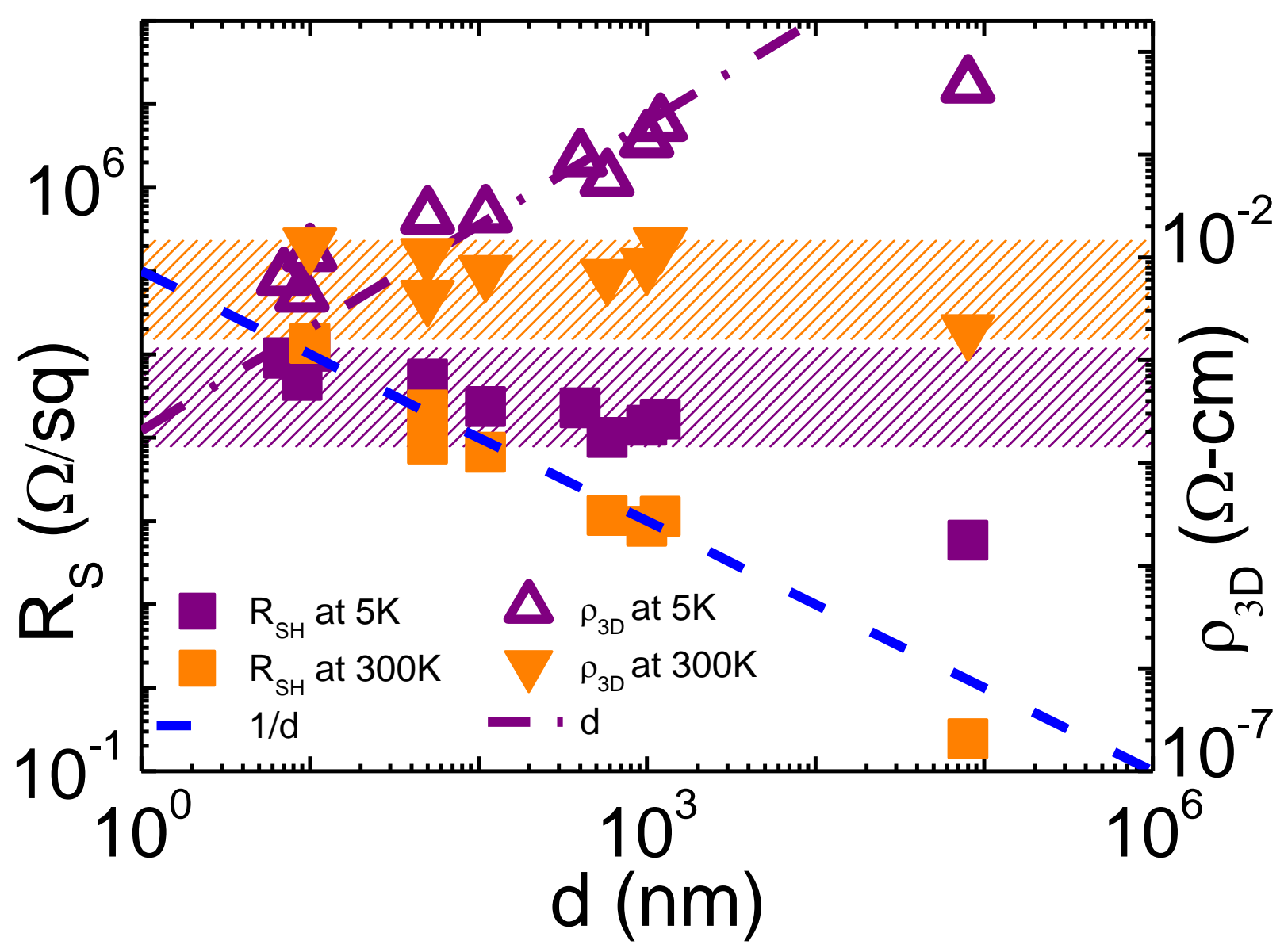

Supplementary Figure S7. Thickness $(d)$ dependence of sheet-resistance $\left(R_{S}\right)$ and resistivity $\left(\rho_{3 D}\right)$ at $5 \mathrm{~K}$ and $300 \mathrm{~K}$. 


\section{Fitting Of $\mathbf{R}_{S}$ vs $\mathbf{V}_{G}$ Data}

For a quantitative treatment (schematized in Fig. 1f), we consider two parallel conducting channels (top and bottom surfaces) in the presence of a back gate voltage. Assuming that both charge impurity scattering (constant mobility $\mu$ ) and short range defect scattering ( $\mu^{s} \propto 1 / n, n$ is number density of the channel) exist independently in same channel, Matthiessen's rule [3] gives, total mobility

$$
\frac{1}{\mu_{t}}=\frac{1}{\mu}+\frac{1}{\mu^{s}}
$$

and conductance,

$$
\begin{aligned}
\sigma & =n e \mu_{t} \\
& =\left(\frac{1}{n e \mu_{t}}\right)^{-1} \\
& =\left[\frac{1}{n e}\left(\frac{1}{\mu}+\frac{1}{\mu^{s}}\right)\right]^{-1} \\
& =\left[\frac{1}{n e}\left(\frac{1}{\mu}+\frac{1}{C / n}\right)\right]^{-1}
\end{aligned}
$$

where $\mu^{s}=C / n, C$ is a constant.

$$
\sigma=\left[\frac{1}{n e} \frac{1}{\mu}+\rho_{s}\right]^{-1}
$$

where $\rho_{S}=1 / C e, \rho_{S}$ is the resistivity due to short range scattering.

Ignoring any contribution from the bulk, the net resistivity of the flake can be written as

$$
\begin{aligned}
R_{s}^{-1} & =\sigma_{b}+\sigma_{t} \\
& =\left[\frac{1}{n_{b} e \mu_{b}}+\rho_{0 b}\right]^{-1}+\left[\frac{1}{n_{t} e \mu_{t}}+\rho_{0 t}\right]^{-1} \\
& =\left[\frac{1}{C_{o x}\left(V_{g}-V_{0 b}\right) \mu_{b}}+\rho_{0 b}\right]^{-1}+\left[\frac{1}{C_{o x}\left(V_{g}-V_{0 t}\right) \mu_{t}}+\rho_{0 t}\right]^{-1}
\end{aligned}
$$

here $n_{b(t)}, \sigma_{b(t)}, \mu_{b(t)}, V_{0 b(t)}$, and $\rho_{0 b(t)}$ are the number density, sheet-conductance, charged impurity-limited mobility, charge neutrality points and sheet-resistance due to short range defect scattering for the bottom (top) surface, respectively. Due to strong coupling of the surfaces, for sample D10 we can assume $V_{0 b}=V_{0 t}=V_{0}([4])$. That leads to the equation

$$
R_{s}^{-1}=\left[\frac{1}{C_{o x}\left(V_{g}-V_{0}\right) \mu_{b}}+\rho_{0 b}\right]^{-1}+\left[\frac{1}{C_{o x}\left(V_{g}-V_{0}\right) \mu_{t}}+\rho_{0 t}\right]^{-1}
$$

Our fit (Fig 1f) shows $\mu_{b}=\mu_{t}=\mu \simeq 70 \mathrm{~cm}^{2} /$ V.s and $\rho_{0 b}=\rho_{0 t}=\rho_{0}=5 \mathrm{k} \Omega / \mathrm{sq}$. The equality of $\rho_{0 b}$ and $\rho_{0 t}$ indicates that the short range scattering is likely to arise from defects that are intrinsic to the TI itself, rather than extraneous factors such as substrate or surface adsorbates which would influence the two surfaces differently. 

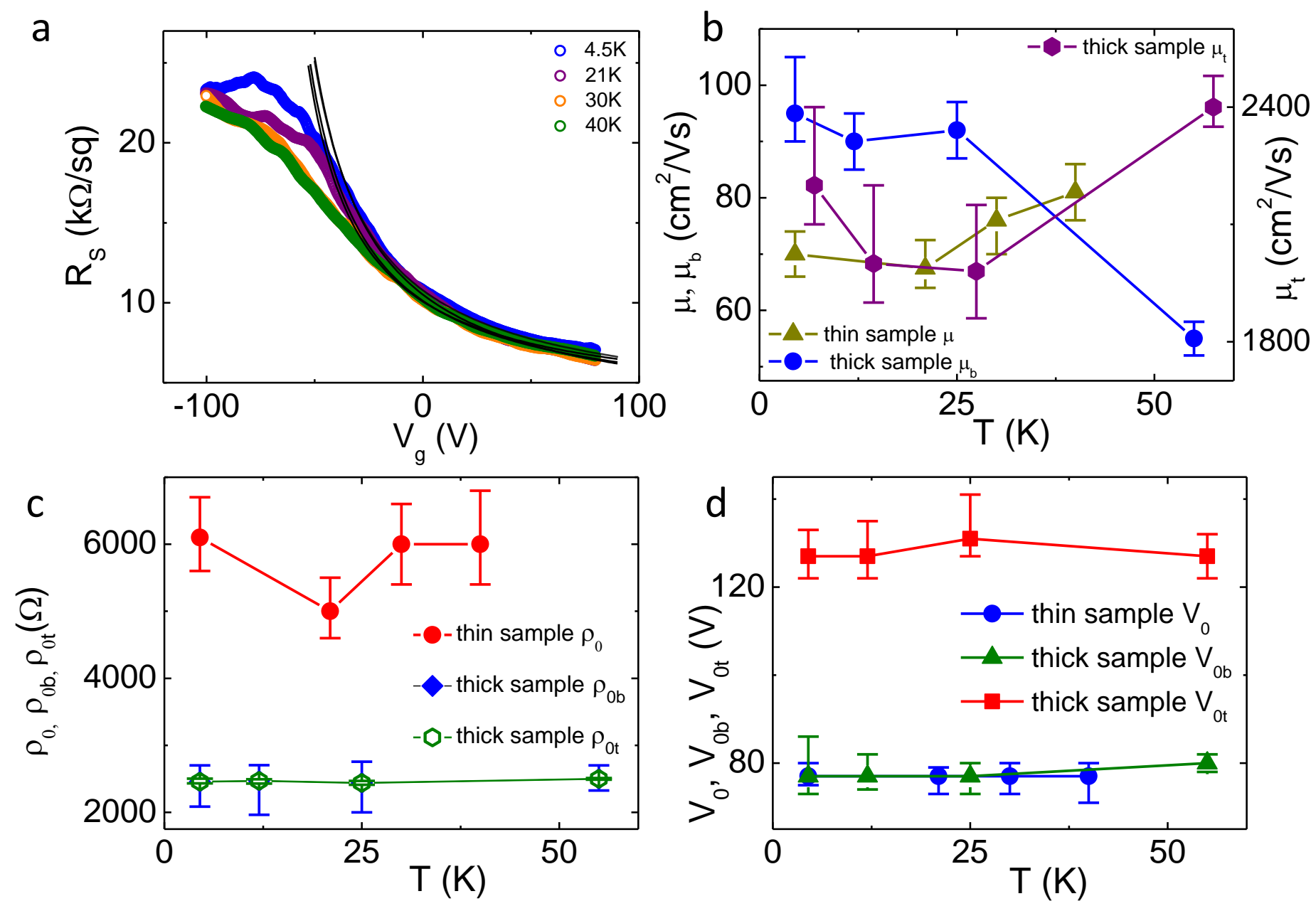

Supplementary Figure S8. (a) $R_{S}$ vs $V_{g}$ data and fit according to Eq. 9 of supplementary information. Fit parameters (b) $\mu, \mu_{b}, \mu_{t}$, (b) $\rho_{0}, \rho_{0 b}, \rho_{0 t}$, (c) $V_{0}, V_{0 b}, V_{0 t}$ as a function of temperature for thick and thin sample.

For fitting the thicker sample D110 we have used Eq. 9 as $V_{0 b} \neq V_{0 t}$ for the reasons already explained in the main text. As the cause of doping is common for bottom surfaces of D10 and D110 we can assume $V_{0 b}=V_{0}$ and take $V_{0 t}$ as a fitting parameter. The resulting fit, showed by the solid lines in Fig. 1e, reveals $\mu_{b} \approx 120 \mathrm{~cm}^{2} / \mathrm{V}$.s, $\mu_{t} \approx 2100 \mathrm{~cm}^{2} /$ V.s and $V_{0 t} \sim-125 \mathrm{~V}$. Smaller value of $\rho_{0 b}=\rho_{0 t} \approx 2.5 \mathrm{k} \Omega$ compared to D10 indicates that the exfoliation process introduces more defects in the thinner samples. 


\section{Sheet-resistance $\left(R_{S}\right)$ vs temperature $(T)$ fit}

Conductance through the surface metallic states $G_{\text {sur }}=\left(R_{0}+A T\right)^{-1}$, where $R_{0}$ is the impurity-scattering limited resistance and $A T$ is the resistance term from electron-phonon scattering.

Conductance through the thermally activated bulk channel $G_{b u l k}=t\left(\rho_{b 0} e^{\Delta E / k_{B} T}\right)^{-1}$, where $t, \rho_{b 0}, \Delta E, k_{B}$ are thickness of the flake, room-temperature bulk-resistivity, activation energy and Boltzman constant.

Assuming parallel channel of conduction through both surfaces and insulating bulk, total areal-Resistance can be fitted with

$$
R_{S}=\left(\left(R_{0}+A T\right)^{-1}+t\left(\rho_{b 0} e^{\Delta E / k_{B} T}\right)^{-1}\right)^{-1}
$$

with $R_{0}, A, \rho_{b 0}$ and $\Delta E$ as fitting parameters.
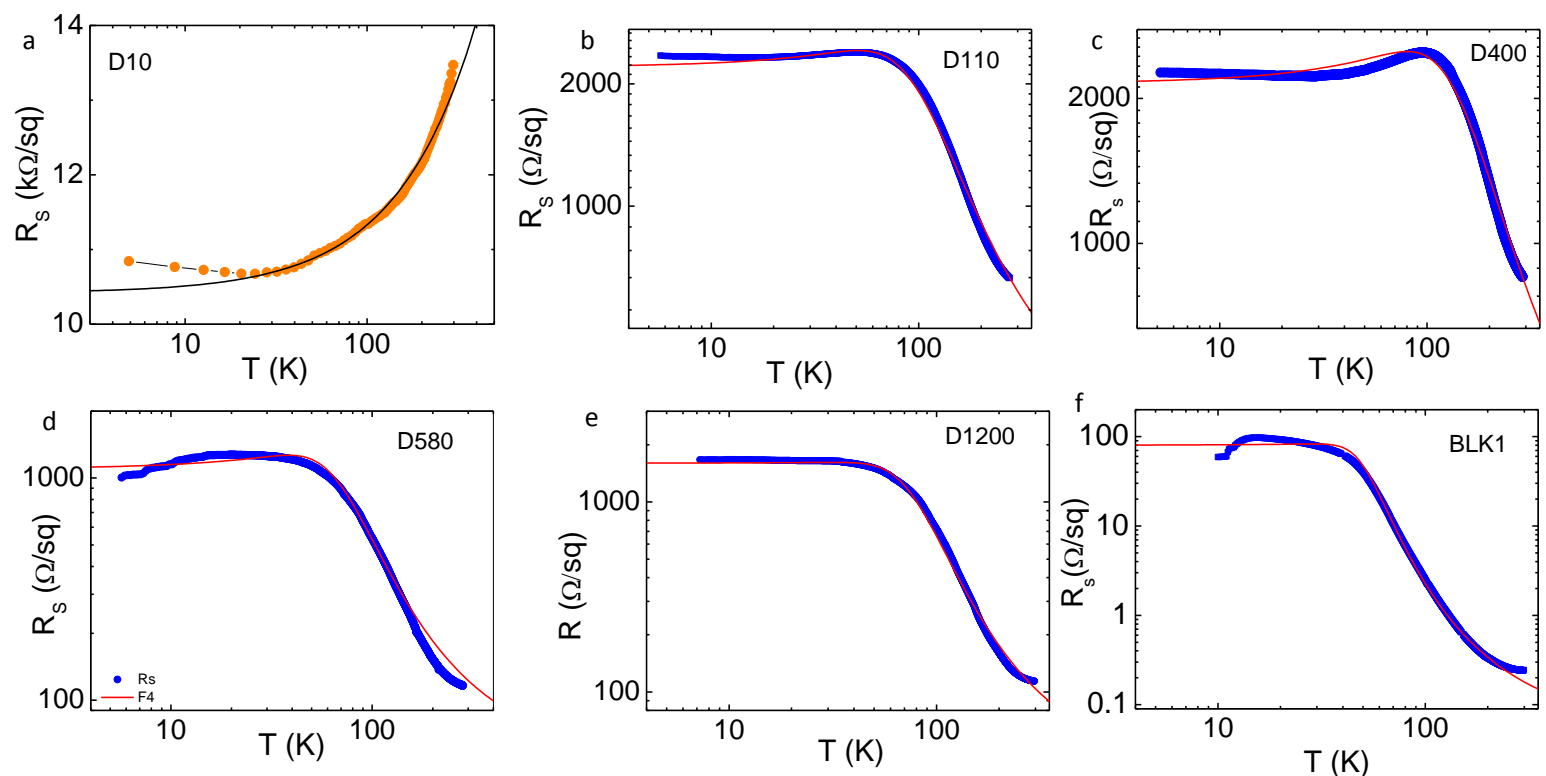

Supplementary Figure S9. $R_{S}$ vs $T$ data and fit according to Eq. 11 of supplementary information.
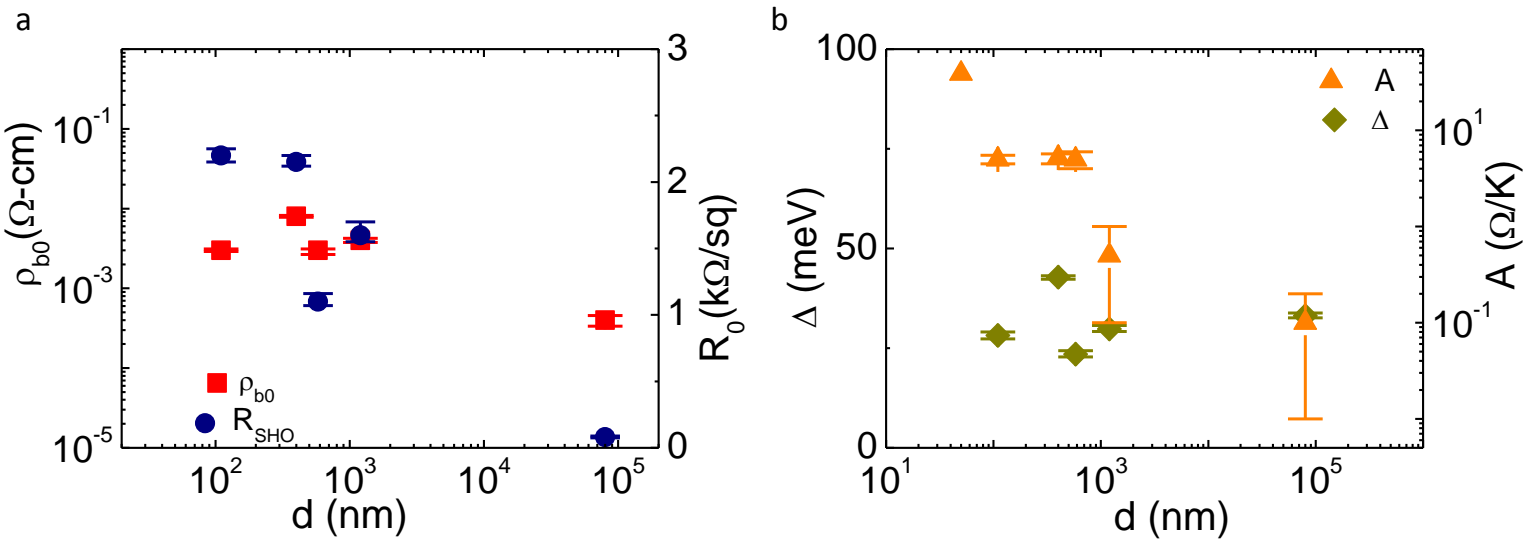

Supplementary Figure S10. Fit parameters from the fits shown in figure S9. 


\section{Fitting Noise data with McWhorter Model}

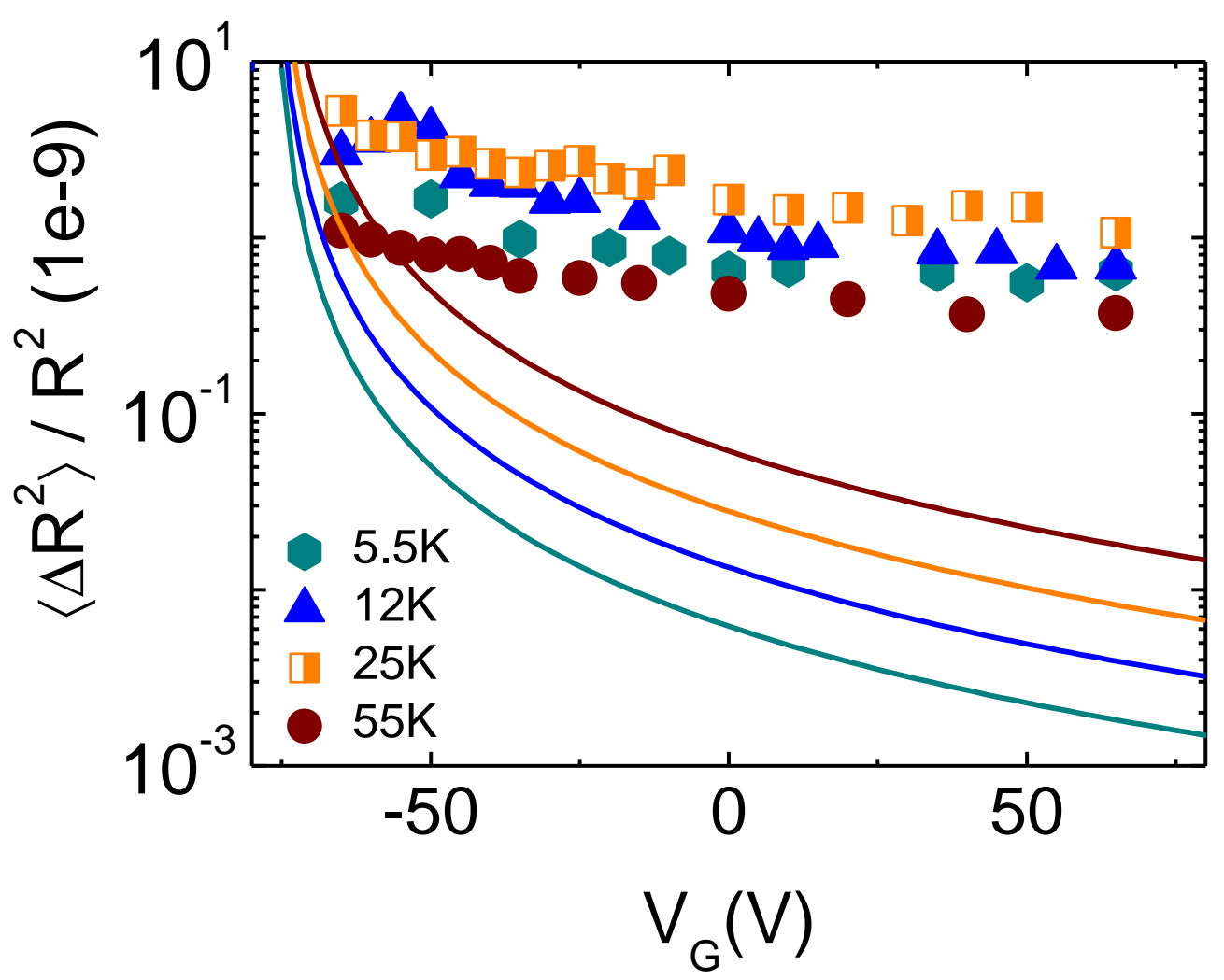

Supplementary Figure S11. Noise vs $V_{g}$ data in D110 sample. The colored continuous lines show Noise as a function of $V_{g}$ according to Eq. 12

Considering McWhorter mechanism for bottom surface,

$$
N_{b}=\frac{M_{C W}}{\left(V_{G}-V_{0}\right)^{2}}
$$

where $M_{c w}=\frac{e^{2} k_{B} T D_{i t}}{A C_{o x}^{2} \alpha} \log \frac{f_{2}}{f_{1}}$ where $A, e, k_{B}, T, D_{i t}, C_{o x}, \alpha, f_{2}$ and $f_{1}$ are area of device, electronic charge, Boltzman constant, temperature, trap density per unit of energy per unit of volume, capacitance of $300 \mathrm{~nm} \mathrm{SiO}_{2}$, spatial decay constant and upper and lower bound of the measured spectrum [5] respectively. The solid lines in supplementary Fig. S11 follow Eq. 12 with typical value of trap density reported in literature $[6]\left(D_{i t}=5.6 \times 10^{43}\right.$ $\left.\mathrm{J}^{-1} \mathrm{~m}^{-3}\right)$. 
IX. Noise as a function of gate voltage at $81 \mathrm{~K}$.

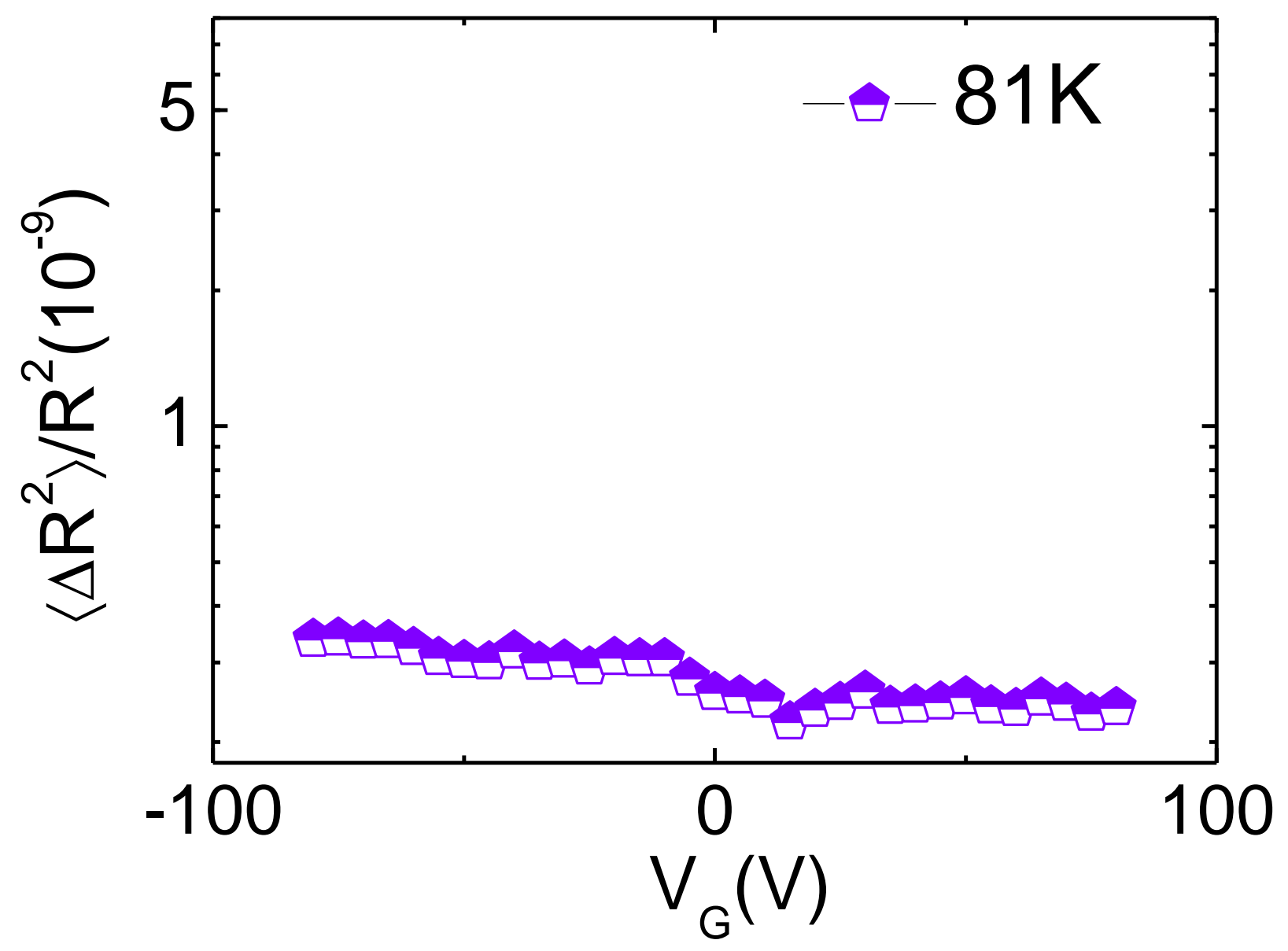

Supplementary Figure S12. Noise as a function of gate voltage in sample D110 at $81 \mathrm{~K}$. 
X. Noise and sheet-resistance vs Temperature in D10.

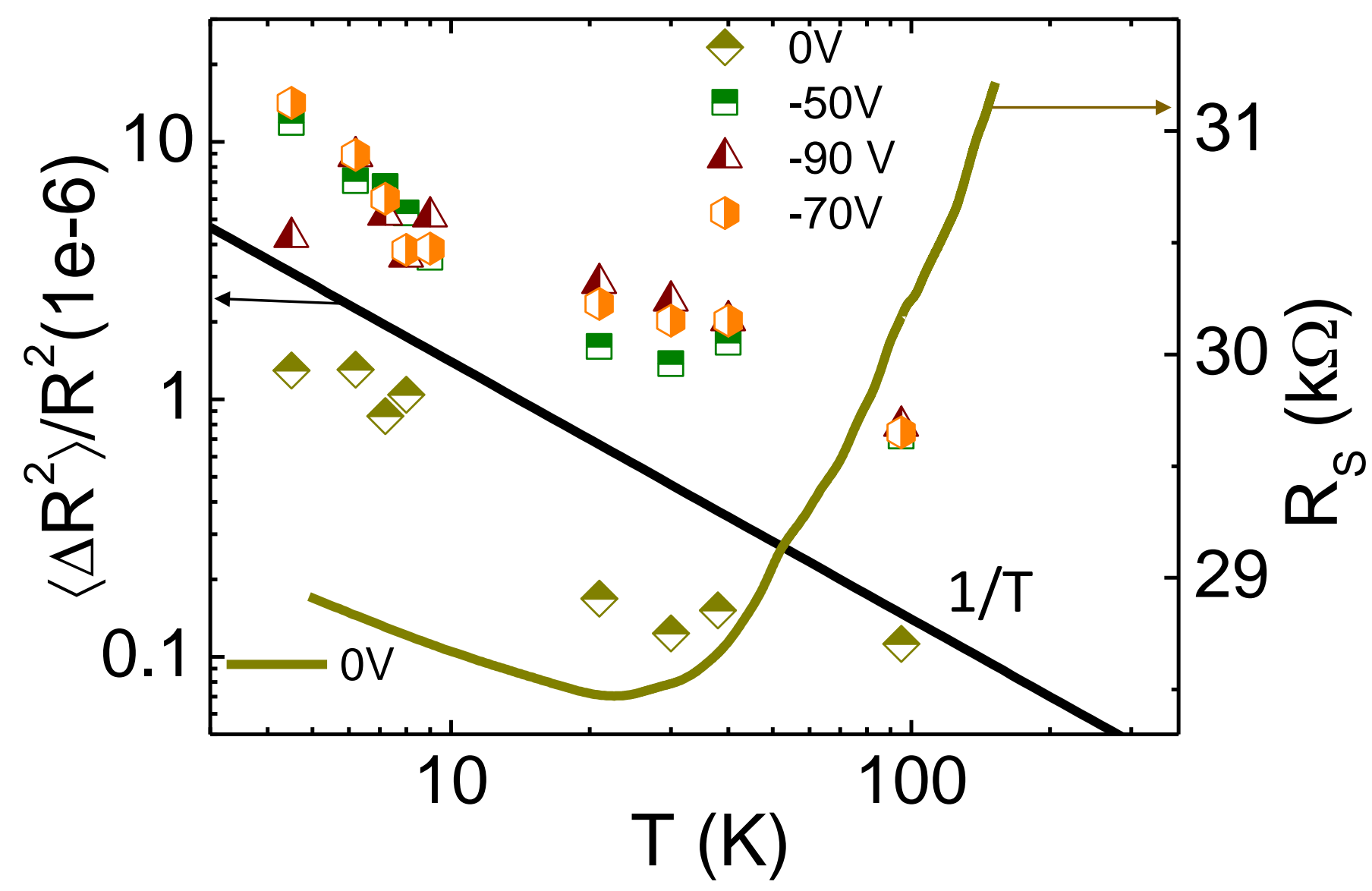

Supplementary Figure S13. Relative variance of resistance $\left(\frac{\left\langle\Delta R^{2}\right\rangle}{R^{2}}\right)$ and sheet-resistance $\left(R_{S}\right)$ as a function of temperature $(T)$ in D10. The solid line shows $1 / T$ type of dependence of noise. 
XI. Noise vs Temperature in D1000.

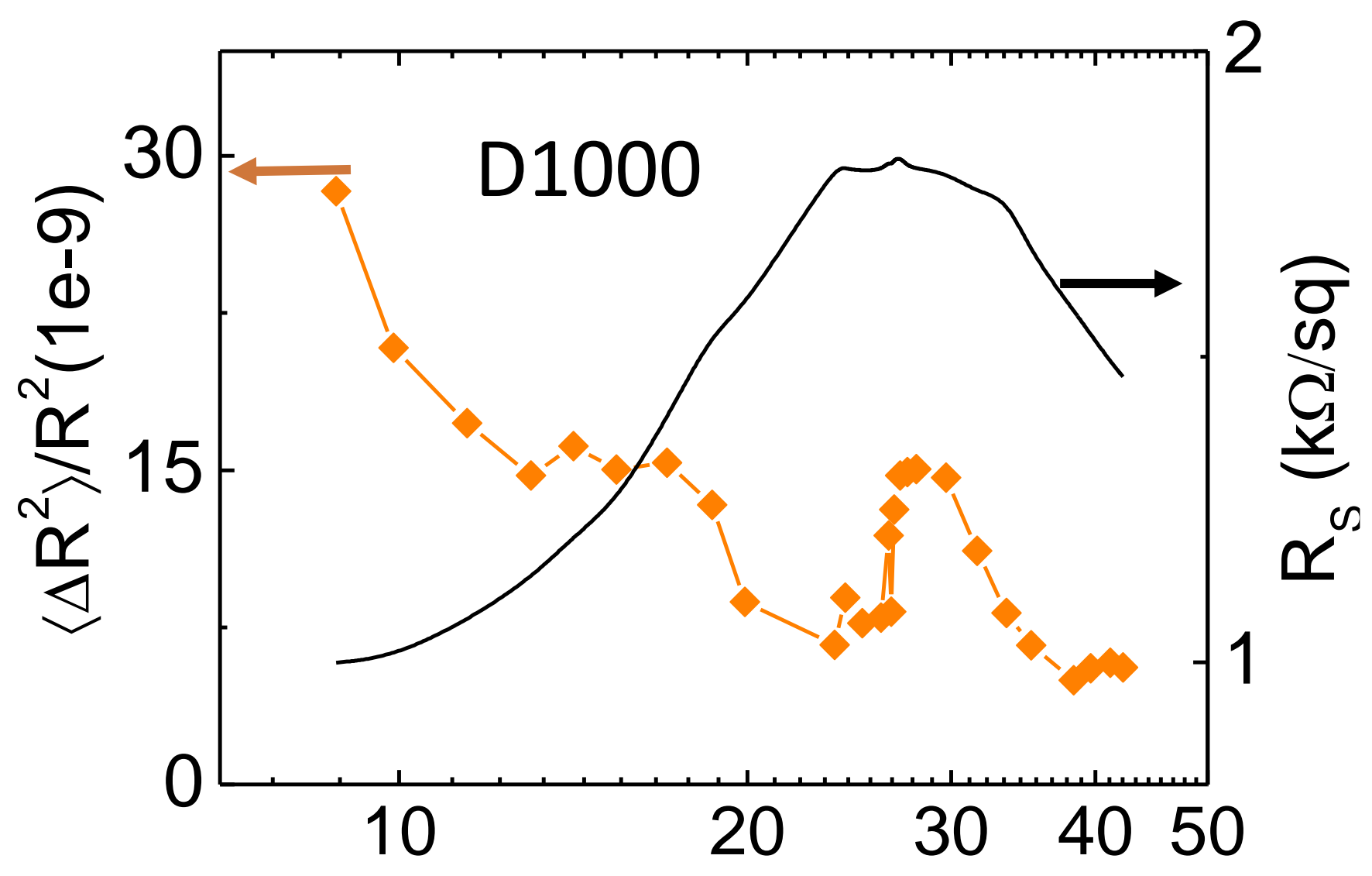

Supplementary Figure S14. Relative variance of resistance $\left(\frac{\left\langle\Delta R^{2}\right\rangle}{R^{2}}\right)$ and sheet-resistance $\left(R_{S}\right)$ as a function of temperature $(T)$ in D1000. 
XII. $f S_{V} / V^{2}$ as a function of $f$ (frequency) in D1000.

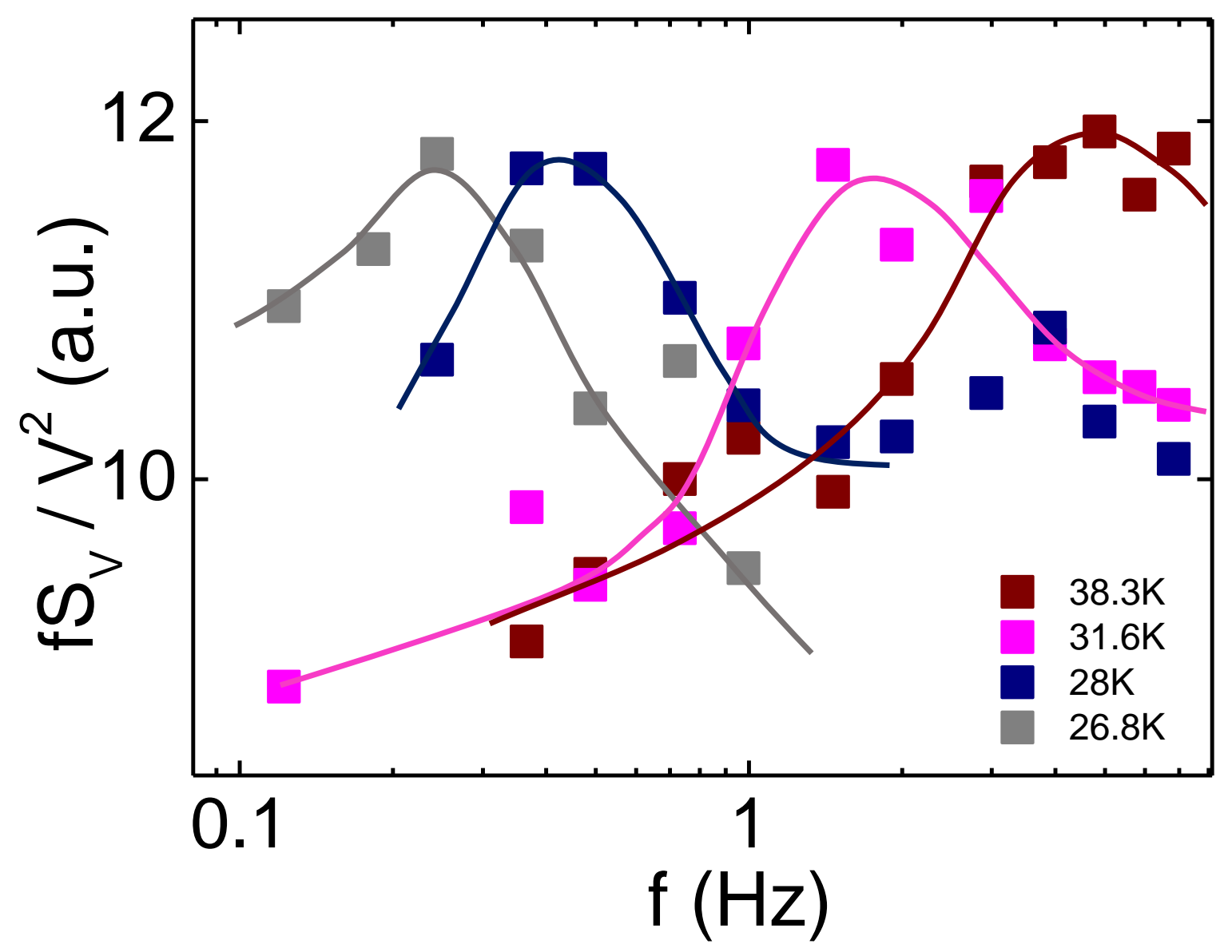

Supplementary Figure S15. $f S_{V} / V^{2}$ as a function of $f$ at different temperatures in the sample D1000. Solid lines are guide to the eye to show the shift of maximum frequency $\left(f_{\max }\right)$ with temperature. 


\section{Hall data in sample D7.}

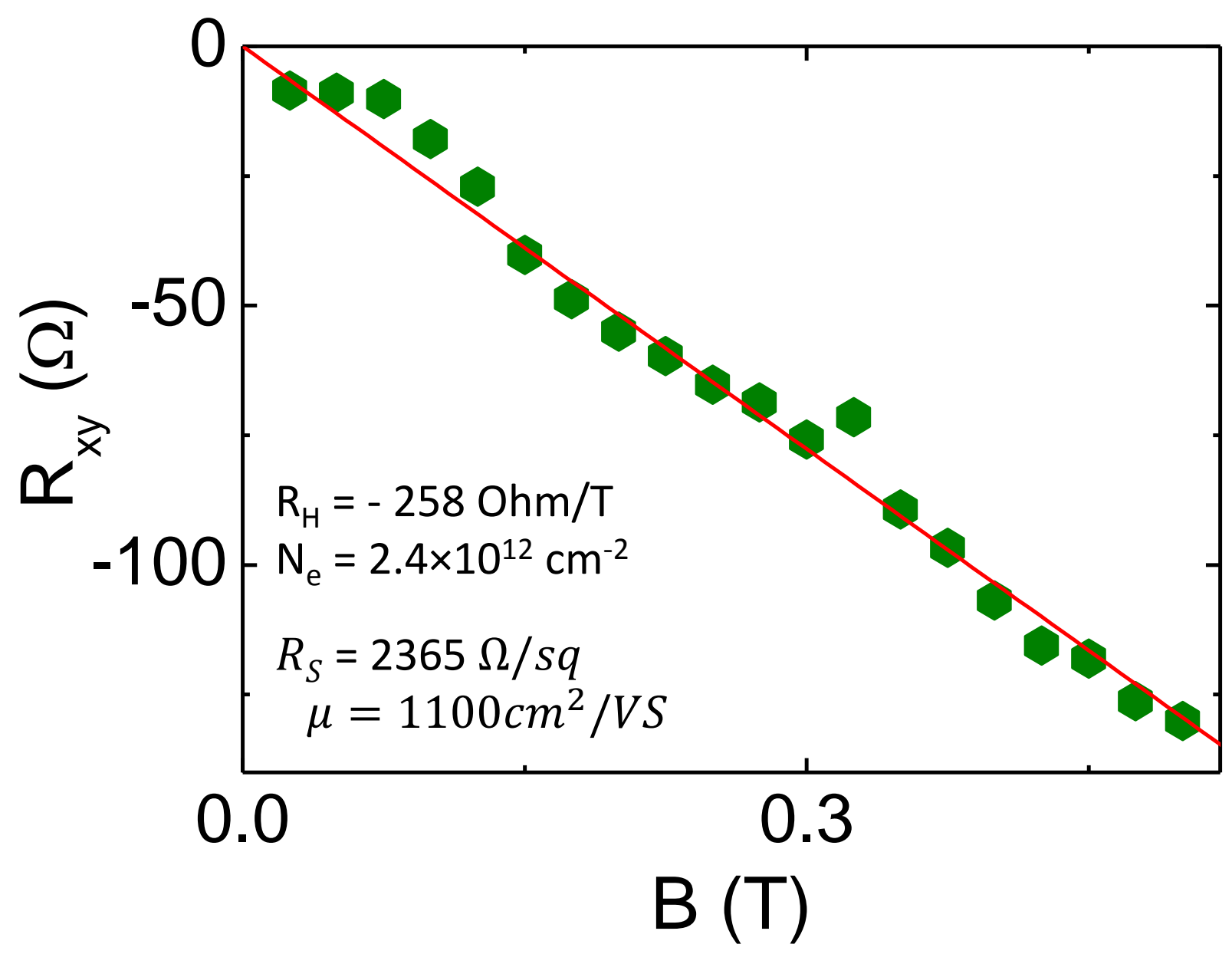

Supplementary Figure S16. Hall measurement in a $7 \mathrm{~nm}$ sample D7.

[1] Ren, Z., Taskin, A. A., Sasaki, S., Segawa, K. \& Ando, Y. Optimizing $\mathrm{Bi}_{2-x} \mathrm{Sb}_{x} \mathrm{Te}_{3-y} \mathrm{Se}_{y}$ solid solutions to approach the intrinsic topological insulator regime. Phys. Rev. B 84, 165311 (2011).

[2] Sokolov, O., Skipidarov, S., Duvankov, N. \& Shabunina, G. Chemical reactions on the $\mathrm{Bi}_{2} \mathrm{Te}_{3}-\mathrm{Bi}_{2} \mathrm{Se}_{3} \mathrm{Section}_{\text {in }}$ the process of crystal growth. Journal of Crystal Growth 262, 442-448 (2004).

[3] Yan, J. \& Fuhrer, M. S. Correlated charged impurity scattering in graphene. Phys. Rev. Lett. 107, 206601 (2011).

[4] Kim, D. et al. Surface conduction of topological Dirac electrons in bulk insulating $\mathrm{Bi}_{2} \mathrm{Se}_{3}$. Nat. Phys. 8, 459-463 (2012).

[5] Jayaraman, R. \& Sodini, C. G. 1/f Noise Technique To Extract the Oxide Trap Density Near the Conduction Band Edge of Silicon. IEEE Transactions on Electron Devices 36, 1773-1782 (1989).

[6] Na, J. et al. Low-frequency noise in multilayer $\mathrm{MoS}_{2}$ field-effect transistors: the effect of high-k passivation. Nanoscale 6, 433-441 (2014). 